\title{
Glitter in the Darkness? Non-fibrillar $\beta$-amyloid Plaque Components Significantly Impact the $\beta$-amyloid PET Signal in Mouse Models of Alzheimer's Disease
}

\author{
Gloria Biechele ${ }^{1 *}$, Laura Sebastian Monasor ${ }^{2,3^{*}}$, Karin Wind ${ }^{1}$, Tanja Blume ${ }^{1,2}$, Samira \\ Parhizkar $^{4}$, Thomas Arzberger ${ }^{2}$, Christian Sacher ${ }^{1}$, Leonie Beyer ${ }^{1}$, Florian Eckenweber ${ }^{1}$, \\ Franz-Josef Gildehaus ${ }^{1}$, Barbara von Ungern-Sternberg ${ }^{1}$, Michael Willem ${ }^{5}$, Peter \\ Bartenstein $^{1,6}$, Paul Cumming ${ }^{7,8}$, Axel Rominger ${ }^{1,7}$, Jochen Herms ${ }^{2,6,9}$, Stefan F. \\ Lichtenthaler ${ }^{2,6,10}$, Christian Haass ${ }^{2,5,6}$, Sabina Tahirovic ${ }^{2^{\star}}$, Matthias Brendel ${ }^{1,6^{*}}$ \\ ${ }^{1}$ Dept. of Nuclear Medicine, University Hospital of Munich, LMU Munich, Munich, Germany \\ ${ }^{2}$ German Center for Neurodegenerative Diseases (DZNE) Munich, Munich, Germany \\ ${ }^{3}$ Graduate School of Systemic Neuroscience, Ludwig-Maximilians-University Munich, Munich, Germany \\ ${ }^{4}$ Dept. of Neurology, Washington University, St. Louis, USA \\ ${ }^{5}$ Chair of Metabolic Biochemistry, Biomedical Center (BMC), Faculty of Medicine, LMU Munich, Munich, \\ Germany \\ ${ }^{6}$ Munich Cluster for Systems Neurology (SyNergy), Munich, Germany \\ ${ }^{7}$ Department of Nuclear Medicine, Inselspital, University Hospital Bern, Bern, Switzerland \\ ${ }^{8}$ School of Psychology and Counselling and IHBI, Queensland University of Technology, Brisbane, \\ Australia \\ ${ }^{9}$ Center of Neuropathology and Prion Research, University of Munich, Munich Germany \\ ${ }^{10}$ Neuroproteomics, School of Medicine, Klinikum Rechts der Isar, Technical University of Munich, Munich, \\ Germany \\ ${ }^{*}$ Contributed equally
}

$19 / 03 / 2021$

Abbreviated title: $\beta$-amyloid PET signal source

Corresponding author: Dr. Matthias Brendel; Department of Nuclear Medicine, University of Munich; Marchioninistraße 15, 81377 Munich, Germany; Phone:+49(0)89440074650, Fax:+49(0)89440077646; EMail: matthias.brendel@med.uni-muenchen.de

First author: Gloria Biechele (medical student); Department of Nuclear Medicine; LMU Munich, Germany; Phone:+49(0)89440074646; E-Mail: gloria.biechele@med.uni-muenchen.de 


\section{ABSTRACT}

Objective: $\beta$-amyloid PET (A $\beta$-PET) is an important tool for quantification of amyloidosis in the brain of suspected Alzheimer's disease (AD) patients and transgenic AD mouse models. Despite the excellent correlation of A $\mathrm{B}-\mathrm{PET}$ with gold standard immunohistochemical assessments, the relative contributions of fibrillar and non-fibrillar $A \beta$ components to the in vivo $A \beta-P E T$ signal remain unclear. Thus, we obtained two murine cerebral amyloidosis models that present with distinct $A \beta$ plaque compositions and performed regression analysis between immunohistochemistry and $A \beta P E T$ to determine the biochemical contributions to $A \beta-P E T$ signal in vivo. Methods: We investigated groups of $A p p^{N L-G-F}$ and APPPS1 mice at three, six and 12 months of age by longitudinal ${ }^{18} \mathrm{~F}$-florbetaben A $\beta$-PET and with immunohistochemical analysis of the fibrillar and total $A \beta$ burdens. We then applied group level inter-modality regression models using age and genotype matched sets of fibrillar/ non-fibrillar $A \beta$ data (predictors) and A $\beta-P E T$ results (outcome) for both transgenic models. An independent group of double-hit APPPS1 mice with dysfunctional microglia due to knock-out of triggering receptor expression on myeloid cells 2 $\left(\right.$ Trem2 $\left.{ }^{-/}\right)$served for validation and evaluation of translational impact. Results: Neither fibrillar nor non-fibrillar $A \beta$ content alone sufficed to explain the $A \beta-P E T$ findings in either transgenic $A D$ model. However, a regression model compiling fibrillar and non-fibrillar $A \beta$ together with the estimate of individual heterogeneity and age at scanning could explain a $93 \%$ of variance of the $A \beta$-PET signal $(P<0.001)$. Fibrillar $A \beta$ burden had a 16 -fold higher contribution to the $A \beta-P E T$ signal when compared to non-fibrillar $A \beta$. However, given the relatively greater abundance of nonfibrillar $A \beta$, we estimate that non-fibrillar $A \beta$ produced $79 \pm 25 \%$ of the net in vivo $A \beta-P E T$ signal in App ${ }^{N L-G-F}$ mice, and $25 \pm 12 \%$ in the APPPS1 mice. Corresponding results in separate groups of APPPS $1 /$ Trem2 $^{-/-}$and APPPS1/Trem $2^{+/+}$mice validated the calculated regression factors and revealed that the altered fibrillarity due to Trem2 knockout impacts the A $\beta-P E T$ signal. Conclusions: Taken together, the in vivo A $\beta$-PET signal derives from the composite of fibrillar and non-fibrillar $A \beta$ plaque components. While fibrillar $A \beta$ has inherently higher PET tracer binding, 
the greater abundance of non-fibrillar $A \beta$ plaque in $A D$ model mice contributes importantly to the PET signal.

Key words: amyloid, fibrillar, non-fibrillar, PET signal, mouse 


\section{INTRODUCTION}

Positron emission tomography for $\beta$-amyloid (A $\beta$-PET) is now widely used for identification and quantification of amyloidosis in the brain of suspected Alzheimer's disease (AD) patients (1), and has been incorporated into the current research framework for diagnostic recommendations in AD (2). Here, the $A \beta$ status (A) identified by PET serves for diagnosis, together with biomarkers for tau $(\mathrm{T})$ and neuronal injury $(\mathrm{N})(2)$. Furthermore, A $\mathrm{A}-\mathrm{PET}$ is used as an inclusion criteria of antiamyloid immunotherapy clinical trials (3), and as a progression biomarker for therapy evaluation in these trials (4). In the preclinical setting, Aß-PET has also become a useful tool for the dynamic assessment of neuropathology in transgenic $A \beta$ mouse models $(5,6)$. Despite the excellent correlation of A $\mathrm{B}-\mathrm{PET}$ with immunohistochemical gold standard assessments of amyloidosis in patients $(7,8)$ and mouse models of $\mathrm{AD}(6,9)$, there has remained an uncertainty about the relative contributions of fibrillar and non-fibrillar $A \beta$ components in plaques to the A $\beta-P E T$ signal in vivo. This research gap needs to be closed as the two forms have differing neurotoxicity, and there is evidence that alterations in AD-related genes like triggering receptor expressed on myeloid cells 2 (TREM2) and Apolipoprotein $E(A P O E)$ alter the net $A \beta$ plaque fibrillarity, which would consecutively bias the relationship between plaque density with A $\mathrm{A}-\mathrm{PET}$ binding in vivo (5).

A human autopsy validated ${ }^{18} \mathrm{~F}$-florbetaben PET study showed preliminary evidence that diffuse plaques may make only a minor contribution to the net A $\beta-P E T$ signal (10). However, autopsy controlled data with ${ }^{18} \mathrm{~F}$-flutemetamol in vivo (11) and comprehensive in vitro data (12) indicated that the binding of that structurally distinct tracer to diffuse plaques also contributes to the net PET signal. Furthermore, our recent preclinical study revealed a discernible A $\beta$-PET signal in $A p p^{N L-G-F}$ mice (13), although this model displays only very limited fibrillar A $\beta$ pathology (14). Therefore, we aimed to quantify the contributions of fibrillar and non-fibrillar plaque components to the $A \beta-P E T$ signal in vivo in $A D$ model mice. 
We recently demonstrated that the $A p p^{N L-G-F}$ and APPPS1 mouse models exhibit differences in $A \beta$ plaque fibrillarity (14), such that a comparative study of these mice could help to determine the effect of fibrillarity on Aß-PET signal in vivo. Thus, we combined a standardized preclinical ${ }^{18} \mathrm{~F}$-florbetaben PET study with immunohistochemical characterization of fibrillar vs. non-fibrillar A $\beta$ in App ${ }^{N L-G-F}$ and APPPS1 mice examined at different pathological stages. We then developed a regression model for immunohistochemistry and A $\mathrm{A}-\mathrm{PET}$ to establish the relative proportions of fibrillar and non-fibrillar sources in the Aß-PET signal in vivo. Furthermore, we validated the calculated regression factors in an independent cohort APPPS1/Trem2 $2^{-/-}$and APPPS1/Trem $2^{+/+}$mice and tested a hypothesis that the non-fibrillar $A \beta$ pool contributes more to the A $\beta-P E T$ signal in APPPS1/Trem2 $2^{-/-}$mice than in APPPS1/Trem2 ${ }^{+/+}$mice.

\section{MATERIAL AND METHODS}

\section{Experimental Design}

All experiments were performed in compliance with the National Guidelines for Animal Protection, Germany and with the approval of the regional animal committee (Regierung Oberbayern), and were overseen by a veterinarian. Animals were housed in a temperature- and humidity-controlled environment with $12 \mathrm{~h}$ light-dark cycle, and with free access to food (Sniff, Soest, Germany) and water. We conducted longitudinal ${ }^{18} \mathrm{~F}$-florbetaben PET imaging in cohorts of female App ${ }^{N L-G-F}(n=18)$ and APPPS1 $(n=14)$ mice Aß-PET at three, six and 12 months of age, together with an age- and sex-matched group of wild-type $(n=8)$ mice. $56 \%$ of the transgenic mice had their baseline examination of at three months of age, and the remaining $44 \%$ of the mice were imaged from six to 12 months of age. All mice of each model originated from the same breeding colony. To exclude batch effects within each modality, we used separate cohorts of mice (14) for immunohistochemistry analyses of fibrillar and non-fibrillar $A \beta$ plaque components in wildtype and AD model mice $(n=3-4)$ at three, six, and 12 months of age. We then applied inter-modality 
regression models to separate the relative contributions of fibrillar and non-fibrillar $A \beta$ plaque components to $A \beta-P E T$ signals in the two transgenic strains.

\section{Animal Models}

APP/PS1 (APPPS1-21) mice show extensive fibrillar A plaque pathology, first evident at $6-8$ weeks of age (15). In contrast, $A p p^{N L-G-F}\left(A p p^{N L-G-F / N L-G-F}\right)$ is a murine model with relatively limited fibrillar $A \beta$ plaque pathology, but showing $A \beta$ PET signal from eight weeks of age in homozygous mice $(16,17)$. Wild-type controls were C57BL/6 mice.

\section{PET Imaging}

PET Data Acquisition, Reconstruction and Post-Processing: For all PET procedures, radiochemistry, data acquisition, and image pre-processing were conducted according to an established, standardized protocol (6). In brief, we obtained ${ }^{18} \mathrm{~F}$-florbetaben A $\beta$-PET recordings (average dose: 12.1 $\pm 1.8 \mathrm{MBq}$ ) with an emission window of 30-60 min after injection.

PET Image Analysis: We performed all analyses using PMOD (version 3.5; PMOD technologies). Normalization of attenuation-corrected emission images to standardized uptake value ratio (SUVr) images was performed using previously validated periaqueductal gray (PAG) (18) and white matter (WM) reference regions for the App ${ }^{N L-G-F}$ and APPPS1 mouse models, respectively (5). We analyzed the wild-type mice separately with both reference regions to serve as controls for the transgenic models. Bilateral neocortical volumes of interest $\left(15 \mathrm{~mm}^{3}\right)$ matching the region of interest in the immunohistochemistry analysis were applied for calculation of $S U V r_{\text {Forebrain/WM }}$ or $S U V r_{\text {Forebrain/PAG. }}$ 


\section{Immunohistochemical Analysis}

Groups of APPPS1 and App NL-G-F mice at an age of three months $(n=4)$, six months $(n=3)$ and 12 months ( $n=4)$ were transcardially perfused with ice cold PBS $(0.1 M)$ followed by $4 \%$ PFA, after cryopreserved in $30 \%$ sucrose. The mouse tissue used for immunohistochemical analysis included some of the APPPS1 and $A p p^{N L-G-F}$ mouse brains utilized in our previous publication (14). All stainings and analyses were performed newly for the purpose of the present study. We collected $30 \mu \mathrm{m}$-thick coronal sections for free-floating immunostaining. We used the 3552 antibody $(1: 5000 ;(19))$ to label total $A \beta$ and Thiazine red $(2 \mu \mathrm{M}$, Sigma) to stain the fibrillar $A \beta .24$ images were acquired in four coronal sections (6 images per section) in regions matching PET using a confocal microscope (20x dry objective, Leica TCS SP5). Given the prominent differences in the levels of fibrillar A $\beta$ between the APPPS1 and App ${ }^{N L-G-F}$ mice, the confocal settings were optimized for each mouse model to acquire the Thiazine red signal. For the three- and six-monthold $A p p^{N L-G-F}$ mice, the "averaging" and "accumulation" confocal functions were set to "2", to better detect the Thiazine red signal. An in-house programmed macro from ImageJ (NIH) was used to analyze the total and fibrillar $A \beta$ coverage.

As a validation analysis, we reanalyzed data from a previous study that included immunohistochemistry markers for fibrillar (x-34) and total $A \beta(3552)$ components of $A \beta$ plaques (5). Immunohistochemistry was obtained from APPPS1xTrem $2^{+/+}$and APPPS1xTrem2 $2^{-/-}$mice (three and six months: $n=4,12$ months: $n=8$ ). A 3 -PET data were analyzed by the processing pipeline described above and at the same time-points for both genotypes (APPPS $1 \times \mathrm{xTrem}^{+/+}$: three months: $n=3$, six and 12 months: $n=10$; APPPS1xTrem2 ${ }^{---:}$three months: $n=7$, six months: $n=9,12$ months: $n=7)$. Furthermore, for validation purpose we obtained $A \beta$ coverage for fibrillar (methoxy-x04 or $x-34$ ) and total $A \beta(3552)$ components at 13 months of age in the PET cohorts. In all datasets, non-fibrillar $A \beta$ was calculated by subtraction of fibrillar $A \beta$ from total $A \beta$ (area- $\%$ nonfibrillar $=$ area- $\%$ total - area- $\%$ fibrillar $)$. 


\section{Statistics}

Graph Pad Prism (Version 8.43, GraphPad Software, LCC) was used for all statistical tests. A $P$-value of less than 0.05 was considered to be significant for rejection of the null hypothesis.

Group Level Analysis: Non-fibrillar $A \beta$, fibrillar $A \beta$, and the Aß-PET z-score were compared between $A p p^{N L-G-F}$ and APPPS1 mice at different ages by an unpaired Student's t-test. Mean values of each of the three read-outs from the App $p^{N L-G-F}$ and APPPS1 groups at different ages were subject to a linear regression analysis. The area between the regression plots served as an index of the potential bias in the estimates of $A \beta$ pathology by $A \beta-P E T$.

Individual Level Analysis: We applied regression models using the A $\beta$-PET z-score of all investigated mice of both models as an outcome variable. Fibrillar $A \beta$ and non-fibrillar $A \beta$ estimates deriving from all age and genotype-matched mouse groups were used as predictors, and heterogeneity of individual mice with respect to PET results and age were used as additional covariates. Here, we defined heterogeneity as the deviation of individual mice of each genotype from their group mean at each time-point. The regression coefficients for fibrillar $A \beta$ and nonfibrillar $A \beta$ were extracted to calculate their relative contributions to the A $\beta-P E T$ signal. Bootstrapping was performed with 1000 random samples.

Validation Analysis: The derived regression coefficients were applied to immunohistochemistry analysis of independent samples of APPPS1/Trem2 $2^{+/+}$and APPPS1/Trem2-/- mice. The predicted A $\beta$-PET z-scores were compared with the actual Aß-PET z-scores in vivo, and the deviation between the predicted and actual A $\beta$-PET z-scores was compared with separate consideration of both plaque components and sole consideration of fibrillar $A \beta$. The bias resulting from consideration only of fibrillar $A \beta$ was calculated as a function of longitudinal changes in the $A \beta-$ PET signal in the contrast of APPPS1/Trem2 $2^{+/+}$and APPPS1/Trem2 $2^{-/-}$mice. 


\section{RESULTS}

\section{Separate Quantification of Fibrillar or Non-fibrillar A $\beta$ Plaque Deposition Fails to Explain the A $\beta$-PET Signal}

First, we performed a direct standardized comparison of non-fibrillar and fibrillar $A \beta$ estimates by immunohistochemistry and A $\beta$-PET between App $p^{N L-G-F}$ and APPPS1 mouse models at different ages. Non-fibrillar $A \beta$ area coverage of $A p p^{N L-G-F}$ mice exceeded that of APPPS1 mice at three and six months of age, whereas APPPS1 mice had higher non-fibrillar A $\beta$ area coverage at 12 months of age (Fig. 1A; Fig. 2). Fibrillar A $\beta$ area coverage was significantly higher in APPPS1 mice than in App ${ }^{N L-G-F}$ mice at all ages studied (Fig. 1B; Fig. 2). Immunohistochemically assessed area coverage values did not differ between the immunohistochemistry cohorts and the PET cohorts at $12 / 13$ months of age (all $P>0.05$, Supplemental Fig. 1). A $\beta$-PET z-scores of $A p p^{N L-G-F}$ and APPPS1 mice are provided and illustrated in Figs. 1C,D. There were no interindividual SUVr differences between mice imaged three times at 3,6 and 12 months when compared to mice only imaged twice at 6 and 12 months (all $P>0.05$, Supplemental Fig. 2). A 3 -PET showed significantly higher standardized differences in APPPS1 mice when compared to App $p^{N L-G-F}$ mice at six and 12 months, whereas there were no significant differences at three months of age (Fig. 1C). Plotting of $A \beta-P E T$ results as a linear function of non-fibrillar or fibrillar $A \beta$ at different ages indicated a mismatch between the two mouse models (Fig. 1E). Plotting fibrillar $A \beta$ as a linear function of nonfibrillar A $\beta$ coverage underpinned that APPPS1 mice had a higher proportion of fibrillar $A \beta$ compared to $A p p^{N L-G-F}$ (Fig. 1E). The comparison of the linear functions of both mouse models (the area transected by the regression lines) indicated that A $\beta$-PET underestimated the proportion of non-fibrillar $A \beta$ in $A p p^{N L-G-F}$ mice (-2.08 z-score units), but overestimated the proportion of fibrillar $A \beta$ in $A p p^{N L-G-F}$ mice (+2.36 z-score units). Thus, neither fibrillar nor non-fibrillar $A \beta$ alone could explain the combined A $\beta$-PET findings. 


\section{Non-fibrillar $A \beta$ Contributes Significantly to the A $\beta$-PET Signal}

Next, we hypothesized that a combined model of non-fibrillar and fibrillar $A \beta$ components could improve the explanation of variance in the Aß-PET signals. To test this, we established a multiple regression model using all available combinations of age and genotype matched A $\beta-P E T$ immuno/histochemistry estimates with inclusion of all App ${ }^{N L-G-F}$ and APPPS1 mice.

Simplified regression models with either fibrillar or non-fibrillar $A \beta$ as predictors of the $A \beta-$ PET z-score explained $50 \%$ and $32 \%$ of the variance in $A \beta-P E T$, respectively (both $P<0.001$, Table 1). Combined consideration of fibrillar $A \beta$ and non-fibrillar $A \beta$ as predictors of the $A \beta-P E T$ Z-score increased the explained variance to $57 \%(P<0.001$, Table 1, Fig. 3A), and fibrillar $\left(\beta=0.563, \quad P=1.11 \mathrm{e}^{-27}\right)$ and non-fibrillar $A \beta\left(\beta=0.309, \quad P=9.38 \mathrm{e}^{-11}\right)$ were both strong and independent predictors of the A $\beta-P E T$ z-score.

A model including fibrillar and non-fibrillar $A \beta$ components with the estimate of individual heterogeneity yielded $85 \%$ explanation of variance of the A 3 -PET signal (Fig. 3B), and further inclusion of age further increased the explanation of variance of the A $\beta$-PET signal (93\%, Fig. 3C). Thus, age-related factors influence importantly immuno/histochemistry and PET signals (i.e. agedependent perfusion or partial volume effects). We considered "fibrillar $x$ non-fibrillar $x$ heterogeneity" and "fibrillar $\mathrm{x}$ non-fibrillar $\mathrm{x}$ heterogeneity $\mathrm{x}$ age" to be the most accurate models and we calculated the mean regression coefficients from these two models to obtain the contributions of fibrillar $A \beta$ and non-fibrillar $A \beta$ to the PET signal. One percent area covered by fibrillar $A \beta$ explained 3.17 PET z-score units and one percent area covered by non-fibrillar $A \beta$ explained 0.20 PET z-score units, thus indicating a 16-fold higher contribution of fibrillar compared to non-fibrillar $A \beta$. The opposite edges of the $95 \%$ confidence intervals, as assessed by bootstrapping, indicated a possible range between 11-fold and 26-fold for the relationship between fibrillary and non-fibrillar contributions to the A $\beta$-PET signal. Application of this multiplicative factors to the direct comparison of group averaged immuno/histochemistry and A $\beta$-PET scores in 
$A p p^{N L-G-F}$ and APPPS1 mice at different ages confirmed the suitability of this model, as indicated by $98 \%$ explanation of the variance using weighted factors, compared to only $84 \%$ for isolated fibrillar and 55\% for non-fibrillar plaque components (Fig. 3D).

\section{Impact of Fibrillar and Non-fibrillar Plaque Components in Mice with Dysfunctional Microglia}

Finally, we validated our results in independent cohorts of APPPS1 mice, and made an additional investigation of the impact of Trem2 deficiency on the A $\beta$-PET signal in these mice, given that Trem2 is a known driver of changes in the plaque fibrillarity. Application of the regression factors to immuno/histochemistry data indicated an excellent prediction of the actual A $\beta-P E T$ signal in independent cohorts of APPPS1/Trem2 ${ }^{-/-}$and APPPS1/Trem2 ${ }^{+/+}$mice (Fig. 4A). APPPS1 mice with Trem2 loss of function showed a higher contribution of non-fibrillar parts plaque components to the A $\mathrm{A}-\mathrm{PET}$ signal $(30 \%$ at three months, $26 \%$ at six months, and $24 \%$ at 12 months) when compared to APPPS1 mice with intact Trem2 (4\% at three months, $15 \%$ at six months, and $21 \%$ at 12 months; Fig. $4 A$ ). A combined consideration of fibrillar $A \beta$ and non-fibrillar $A \beta$ predicted the actual PET signal more precisely that did sole consideration of fibrillar A (Fig. 4B). Previously calculated increases in $A \beta-P E T$ signal with age in these mice indicated a considerable bias when considering only the fibrillar $A \beta$ component (Supplemental Fig. 3). In summary, microglial dysfunction altered the relative proportions of fibrillar and non-fibrillar $A \beta$, thus directly influencing the $A \beta-P E T$ signal as a function of mouse age.

\section{DISCUSSION}

We provide the first in vivo analysis to elucidate the contributions of fibrillar and non-fibrillar plaque components to the A $\beta$-PET signal. Our data clearly show that non-fibrillar plaque fractions 
have a significant impact on the net ${ }^{18} \mathrm{~F}$-florbetaben binding to $A \beta$ plaques of $A \beta$ mouse models in vivo. Although the resulting A $\beta$-PET tracer signal is inherently 16 -fold higher when comparing equal amounts of fibrillar and non-fibrillar $A \beta$, the larger proportions of non-fibrillar plaque components counterbalance the net contribution. We validated our regression model in an independent cohort of APPPS1 mice, and extended the potential translational impact of our findings by showing that microglial dysfunction can influence the longitudinal A $\beta-P E T$ signal via changing the relative proportions of fibrillar to non-fibrillar plaque components.

In various analyses of single amyloidosis mouse models, there was a strong agreement between $A \beta$-PET and different immuno/histochemistry markers for $A \beta(9,20)$. It is widely acknowledged that the A $\beta$-PET signal with ${ }^{18} \mathrm{~F}$-florbetaben primarily derives from aggregated fibrillar $A \beta$, since this and other clinically approved A $\beta$-PET tracers were derived from the chemical scaffold of thioflavin-T, which only colors fibrillar $A \beta(21)$. However, a recent small animal A $\beta-P E T$ investigation from our lab (18) indicated that there could be discrepancies between immuno/histochemistry and in vivo A $\beta-P E T$ signals, if one attributes the entire PET signal to fibrillar $A \beta$. In fact, $A p p^{N L-G-F}$ mice exhibited an only moderate A $\beta-P E T$ signal, although their plaques were mainly composed of non-fibrillar $A \beta$ (14). Therefore, we applied in this study a standardized ${ }^{18} \mathrm{~F}$-florbetaben PET examination comparing $A p p^{N L-G-F}$ and APPPS1 mice in conjunction with combined histochemical and immunohistochemical examination to elucidate the separate contributions of fibrillar and non-fibrillar $A \beta$ sources to the in vivo A $\beta-P E T$ signal. We performed PET acquisitions in both $A \beta$ models and wild-type mice with identical housing conditions and using the same tomograph and image reconstruction parameters, thus minimizing the potential methodological bias. Nonetheless, we acknowledge that scanning of mice on different days of the week and social hierarchy factors, along with technical factors due to different cage positions might still impact the detection of plaque pathology by A $\beta$-PET. We had to choose between conducting longitudinal PET examination and immuno/histochemistry analysis in separate groups (cross-sectional design), rather than conducting the study in which PET 
examination directly preceded immunohistochemistry in the same mice. Since animal batch effects may introduce a bias into cross-sectional PET quantifications between different ages of a given mouse model, we elected to conduct longitudinal PET imaging together with cross sectional immuno/histochemistry to exclude batch effects, at least for PET. To account further for the heterogeneity (22) and asymmetry (13) of amyloidosis in individual mice of a lineage, we used a bilateral target in a regression model including each available combination of PETimmuno/histochemistry results for each model at each of three ages, controlled for the individual heterogeneity. Regression coefficients for the proportions of fibrillar and non-fibrillar $A \beta$ in different models were robust, and revealed that fibrillar $A \beta$ makes an intrinsically 16 -fold higher contribution to the $A \beta-P E T$ signal when compared to non-fibrillar $A \beta$ in the studied $A \beta$ mouse models. Our preclinical in vivo results concur with the post-mortem validation of human ${ }^{18} \mathrm{~F}$-flutemetamol PET data, where ligand binding to diffuse plaques was the most likely explanation for positive in vivo signals in patients who later proved to have only sparse neuritic plaques at autopsy $(11,23)$. The same research group recently validated the contributions of diffuse and neuritic plaques to the ${ }^{18} \mathrm{~F}$ flutemetamol and ${ }^{11} \mathrm{C}-\mathrm{PiB}$ autoradiography signals in vitro study (12). However, our study comprises the first translation of such findings into the in vivo setting, and enables the arithmetic conversion of $A \beta-P E T$ signals into fibrillar and non-fibrillar $A \beta$ components. We note that structure of the stilbene ${ }^{18} \mathrm{~F}$-florbetaben is different to the structures of the benzothiazoles ${ }^{18} \mathrm{~F}$-flutemetamol and ${ }^{11} \mathrm{C}-\mathrm{PiB}$, which could result in different proportions of fibrillar and non-fibrillar binding capacities in vivo. As usual, the limited resolution of small animal PET systems in relation to the mouse brain size and resulting partial volume effects present a limitation for the transfer of present findings into the human context and we want to emphasize that detailed regression factors cannot be transferred directly. Yet, the demonstration of an inherently 16 -fold higher contribution of fibrillar plaque to the PET signal is concurs with a biophysical chemistry study investigating binding mechanisms of $A \beta$ ligands by molecular docking, molecular dynamics and generalized Bornbased free energy calculations (24). Here, core sites of $A \beta$ fibrils, which are more abundant in 
fibrillar components of the plaque, dominated over surface sites in producing the A $\beta$-PET signal (24).

Our findings could be of translational relevance since $A \beta$-immunotherapy and other treatment strategies against $A D$ may change the proportions of fibrillar and non-fibrillar plaque components, and thus bias the A $\beta$-PET outcome. Furthermore, alterations of microglial genes are associated with changes in plaque morphology, which consequently influence the A $\beta$-PET signal (5). We can correlate the more diffuse amyloid plaque morphology in App ${ }^{N L-G-F}$ mice with differences in plaque morphology observed in AD mice deficient for TREM2 or APOE $(5,25)$. While loss of function mutations of Trem2 are rare in humans, microglia genes seem in general (26) to have a high impact on AD pathology, and modulating microglial function is being intensively studied as a therapeutic strategy for AD (27). Another limitation for the direct translation of our findings towards human $A D$ consists in different binding site densities of $A \beta$ plaques in $A \beta$ mouse models when compared to sporadic AD (28). Thus, comparisons of AB-PET signal intensity between rodents and humans need to be considered with caution. However, the regression model generated in this study should increase the awareness for an impact of non-fibrillar $A \beta$ to the $A \beta$ PET signal in both species. Thus, a potential shift in the plaque proportions needs to be considered when designing future studies with assessment of $A \beta$ load in vivo upon therapeutic modifications that target microglia. Assessment of fibrillar and non-fibrillar plaque components and the respective $A \beta-P E T$ tracer binding properties in autopsy cases after/during disease modifying treatment studies of $A D$ could serve to test the impact of our findings in the human situation.

\section{CONCLUSION}

The A $\beta$-PET signal with ${ }^{18} \mathrm{~F}$-florbetaben in vivo arises from a combination of fibrillar and non-fibrillar plaque components. Fibrillar $A \beta$ has inherently higher tracer binding, but the greater proportion of non-fibrillar $A \beta$ relative to fibrillar $A \beta$ in most plaques mean that the non-fibrillar signal 
source is a relevant component of the total signal. Since experimental $A D$ therapy regimens can shift the proportion of fibrillar vs. non-fibrillar $A \beta$, any longitudinal changes in A $\beta$-PET signal as a read-out of therapy monitoring must be interpreted with caution; a detailed understanding of the biochemical basis of $A \beta-P E T$ signal is critical for the correct use of PET for monitoring novel $A D$ therapies.

\section{DISCLOSURE}

C.H. collaborates with Denali Therapeutics, participated on one advisory board meeting of Biogen, and received a speaker honorarium from Novartis and Roche. C.H. is chief advisor of ISAR Bioscience. P.B., A.R. and M.B. received speaking honoraria from Life Molecular Imaging and GE healthcare. M.B. is an advisor of Life Molecular Imaging. No other potential conflicts of interest relevant to this article exist.

\section{ACKNOWLEDGEMENTS}

We thank Mathias Jucker for providing the APPPS1 mice and to Takashi Saito and Takaomi C.

Saido for providing the App $p^{N L-G-F m i c e . ~ S . T . ~ w a s ~ s u p p o r t e d ~ b y ~ t h e ~ A l z h e i m e r-F o r s c h u n g-I n i t i a t i v e ~}$ e.V (grant number 18014). This work was supported by an Alzheimer's Association Grant through the AD Strategic Fund (ADSF-21-831226-C). This work was supported by the German Research Foundation within the framework of the Munich Cluster for Systems Neurology (EXC 2145 SyNergy/ ID 390857198). 


\section{KEY POINTS}

QUESTION: Does non-fibrillar $\beta$-amyloid $(A \beta)$ contribute to the in vivo $A \beta-P E T$ signal?

PERTINENT FINDINGS: Fibrillar $A \beta$ has 16-fold higher tracer binding, but the greater proportion of non-fibrillar $A \beta$ relative to fibrillar $A \beta$ in most plaques mean that the non-fibrillar signal source is a relevant component of the total A $\mathrm{A}-\mathrm{PET}$ signal.

TRANSLATIONAL IMPLICATIONS: Shifts of the proportion of fibrillar vs. non-fibrillar A $\beta$ need to be considered when interpreting the longitudinal Aß-PET signal for monitoring of therapeutic effects. 


\section{REFERENCES}

1. Koscik RL, Betthauser TJ, Jonaitis EM, et al. Amyloid duration is associated with preclinical cognitive decline and tau PET. Alzheimers Dement (Amst). 2020;12:e12007.

2. Jack CR, Jr., Bennett DA, Blennow $K$, et al. NIA-AA Research Framework: Toward a biological definition of Alzheimer's disease. Alzheimers Dement. 2018;14:535-562.

3. Wessels AM, Tariot PN, Zimmer JA, et al. Efficacy and Safety of Lanabecestat for Treatment of Early and Mild Alzheimer Disease: The AMARANTH and DAYBREAK-ALZ Randomized Clinical Trials. JAMA Neurol. 2020;77:199-209.

4. Sevigny J, Chiao $P$, Bussière $T$, et al. The antibody aducanumab reduces $A \beta$ plaques in Alzheimer's disease. Nature. 2016;537:50-56.

5. Parhizkar S, Arzberger T, Brendel M, et al. Loss of TREM2 function increases amyloid seeding but reduces plaque-associated ApoE. Nat Neurosci. 2019;22:191-204.

6. Overhoff $F$, Brendel $M$, Jaworska A, et al. Automated Spatial Brain Normalization and Hindbrain White Matter Reference Tissue Give Improved [(18)F]-Florbetaben PET Quantitation in Alzheimer's Model Mice. Front Neurosci. 2016;10:45.

7. Sabri O, Sabbagh MN, Seibyl J, et al. Florbetaben PET imaging to detect amyloid beta plaques in Alzheimer's disease: phase 3 study. Alzheimers Dement. 2015;11:964-974.

8. Curtis C, Gamez JE, Singh U, et al. Phase 3 trial of flutemetamol labeled with radioactive fluorine 18 imaging and neuritic plaque density. JAMA Neurol. 2015;72:287-294.

9. Rominger A, Brendel M, Burgold S, et al. Longitudinal assessment of cerebral b-amyloid deposition in mice overexpressing Swedish mutant b-amyloid precursor protein using 18F-florbetaben PET. J Nucl Med. 2013;54:1127-1134.

10. Catafau AM, Bullich S, Seibyl JP, et al. Cerebellar Amyloid-beta Plaques: How Frequent Are They, and Do They Influence 18F-Florbetaben SUV Ratios? J Nucl Med. 2016;57:1740-1745.

11. Ikonomovic MD, Buckley $\mathrm{CJ}$, Heurling $\mathrm{K}$, et al. Post-mortem histopathology underlying betaamyloid PET imaging following flutemetamol F 18 injection. Acta Neuropathol Commun. 2016;4:130.

12. Ikonomovic MD, Buckley CJ, Abrahamson EE, et al. Post-mortem analyses of PiB and flutemetamol in diffuse and cored amyloid-beta plaques in Alzheimer's disease. Acta Neuropathol. 2020;140:463-476. 
13. Sacher C, Blume T, Beyer L, et al. Asymmetry of Fibrillar Plaque Burden in Amyloid Mouse Models. J Nucl Med. 2020;61:1825-1831.

14. Sebastian Monasor L, Muller SA, Colombo AV, et al. Fibrillar Abeta triggers microglial proteome alterations and dysfunction in Alzheimer mouse models. Elife. 2020;9.

15. Radde $\mathrm{R}$, Bolmont $\mathrm{T}$, Kaeser $\mathrm{SA}$, et al. Abeta42-driven cerebral amyloidosis in transgenic mice reveals early and robust pathology. EMBO Rep. 2006;7:940-946.

16. Masuda A, Kobayashi Y, Kogo N, Saito T, Saido TC, Itohara S. Cognitive deficits in single App knockin mouse models. Neurobiol Learn Mem. 2016;135:73-82.

17. Saito T, Matsuba Y, Mihira N, et al. Single App knock-in mouse models of Alzheimer's disease. Nat Neurosci. 2014;17:661-663.

18. Sacher C, Blume T, Beyer L, et al. Longitudinal PET Monitoring of Amyloidosis and Microglial Activation in a Second-Generation Amyloid-beta Mouse Model. J Nucl Med. 2019;60:1787-1793.

19. Yamasaki A, Eimer S, Okochi M, et al. The GxGD motif of presenilin contributes to catalytic function and substrate identification of gamma-secretase. J Neurosci. 2006;26:3821-3828.

20. Poisnel G, Dhilly M, Moustie O, et al. PET imaging with [18F]AV-45 in an APP/PS1-21 murine model of amyloid plaque deposition. Neurobiol Aging. 2012;33:2561-2571.

21. Mathis CA, Mason NS, Lopresti BJ, Klunk WE. Development of positron emission tomography betaamyloid plaque imaging agents. Semin Nucl Med. 2012;42:423-432.

22. Brendel $M$, Jaworska $A$, Herms J, et al. Amyloid-PET predicts inhibition of de novo plaque formation upon chronic gamma-secretase modulator treatment. Mol Psychiatry. 2015;20:1179-1187.

23. Ikonomovic MD, Fantoni ER, Farrar G, Salloway S. Infrequent false positive [(18)F]flutemetamol PET signal is resolved by combined histological assessment of neuritic and diffuse plaques. Alzheimers Res Ther. 2018;10:60.

24. Murugan NA, Halldin C, Nordberg A, Langstrom B, Agren H. The Culprit Is in the Cave: The Core Sites Explain the Binding Profiles of Amyloid-Specific Tracers. J Phys Chem Lett. 2016;7:3313-3321.

25. Ulrich JD, Ulland TK, Mahan TE, et al. ApoE facilitates the microglial response to amyloid plaque pathology. J Exp Med. 2018;215:1047-1058. 
26. Sierksma A, Lu A, Mancuso R, et al. Novel Alzheimer risk genes determine the microglia response to amyloid-beta but not to TAU pathology. EMBO Mol Med. 2020;12:e10606.

27. Lewcock JW, Schlepckow K, Di Paolo G, Tahirovic S, Monroe KM, Haass C. Emerging Microglia Biology Defines Novel Therapeutic Approaches for Alzheimer's Disease. Neuron. 2020;108:801-821.

28. Klunk WE, Lopresti BJ, Ikonomovic MD, et al. Binding of the positron emission tomography tracer Pittsburgh compound-B reflects the amount of amyloid-beta in Alzheimer's disease brain but not in transgenic mouse brain. J Neurosci. 2005;25:10598-10606. 

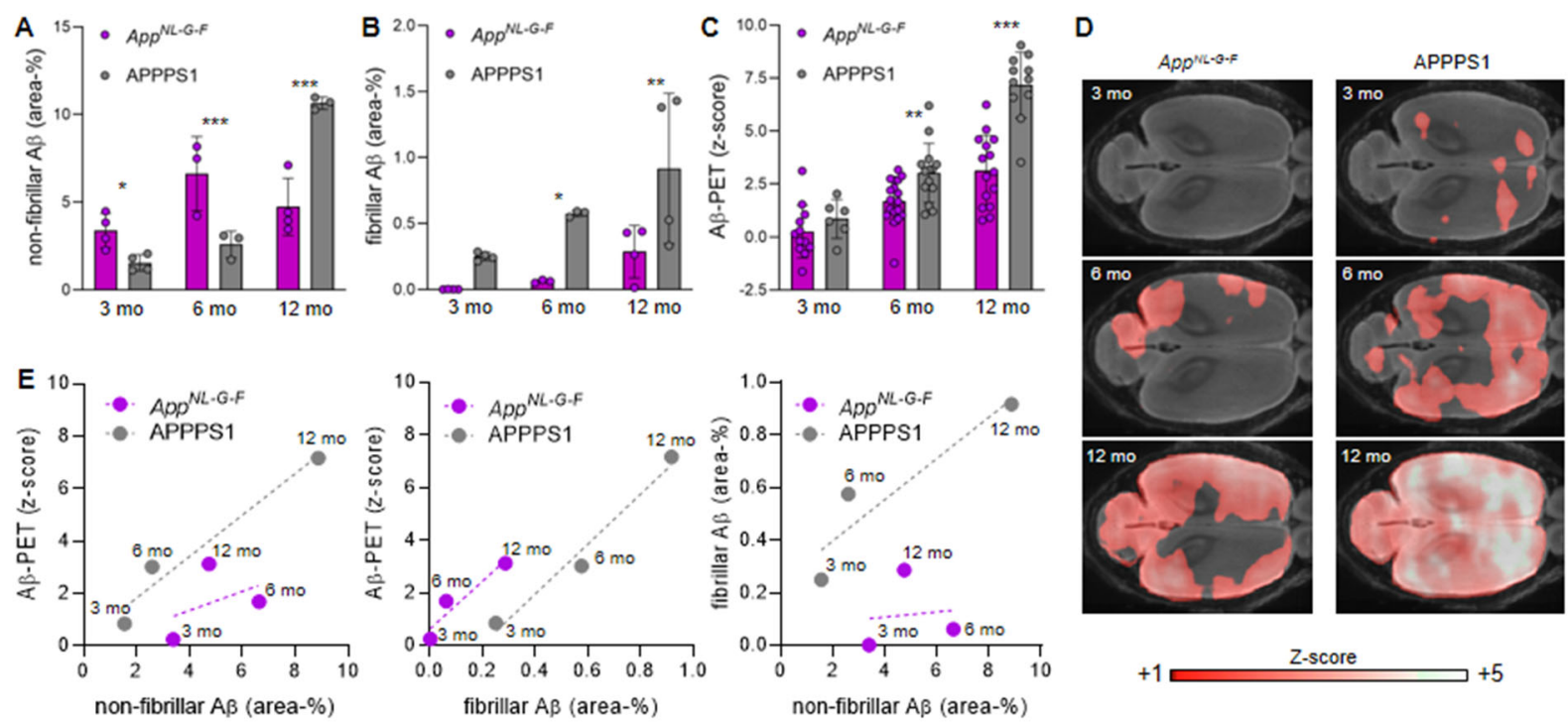

Figure 1: Quantitation of non-fibrillar $A \beta(\mathbf{A})$, fibrillar $A \beta(B)$, and the $A \beta-P E T$ signal z-scores $(\mathbf{C})$ in the neocortex of $A p p^{N L-G-F}$ and APPPS1 mice at three, six and 12 months of age, together with (D) axial images of group-wise PET z-scores projected upon a MRI standard template. (E) Correlation plots of the associations between immuno/histochemistry markers and PET at different ages (group level) in the contrast of both mouse models. ${ }^{*} P<0.05 ;{ }^{* *} P<0.01 ;{ }^{* *} P<0.001$. 


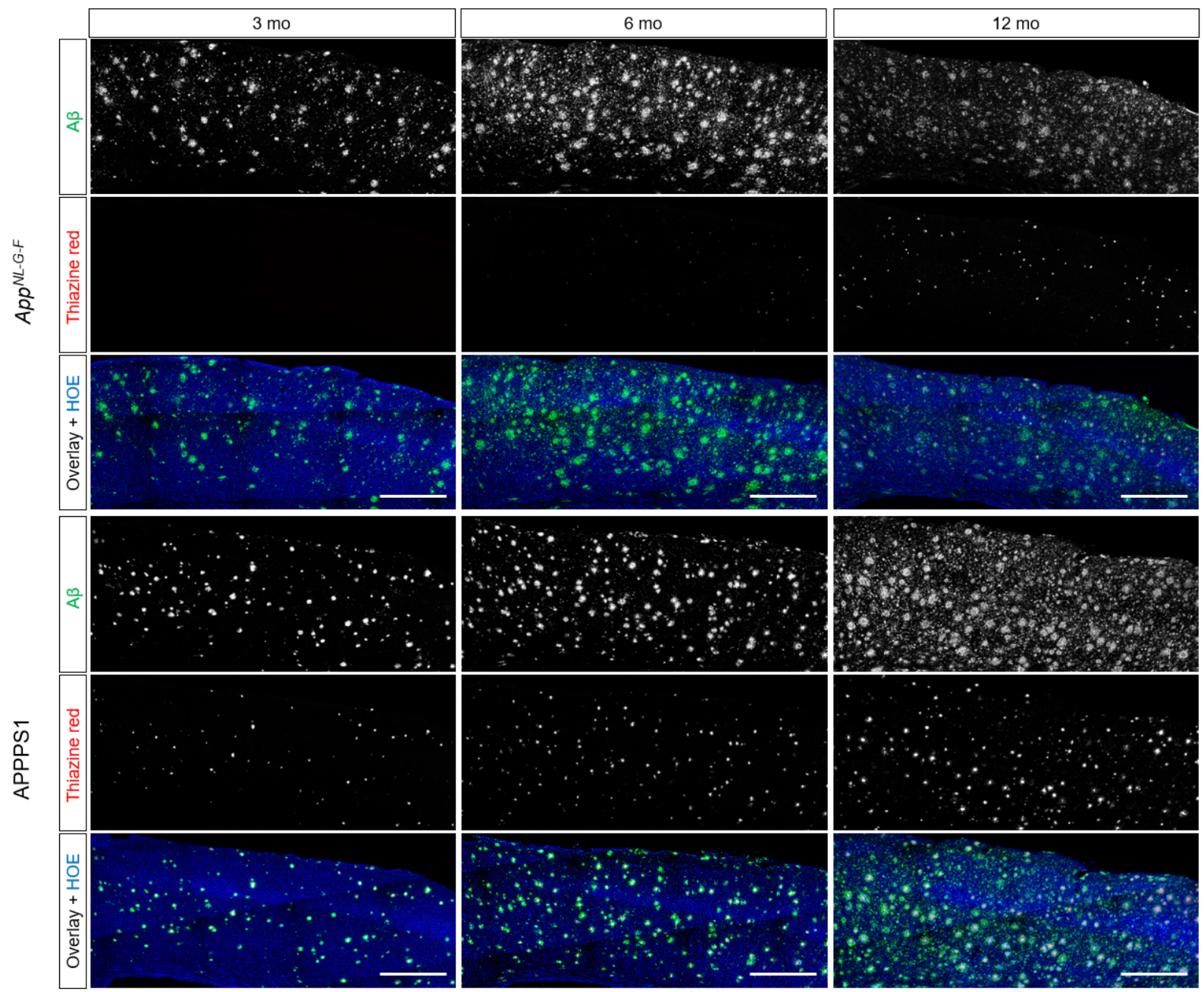

Figure 2: Representative images of immuno/histochemistry. Total $A \beta$ was assessed by 3552 staining and fibrillar $A \beta$ was assessed by Thiazine red. Hoechst (HOE, blue) was used for nuclear visualization. Scale bars: $500 \mu \mathrm{m}$. 

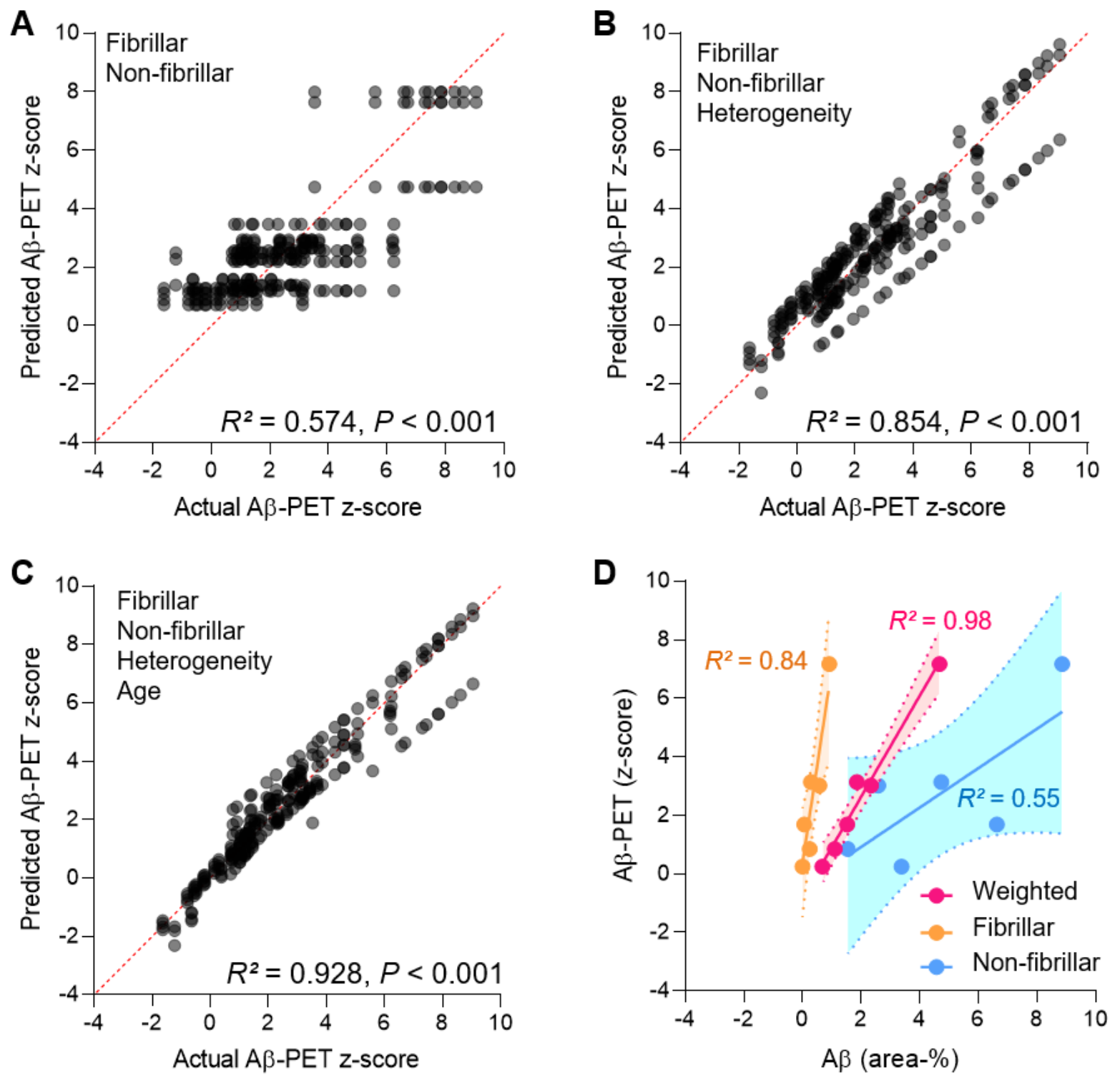

Figure 3: (A-C) Regression plots illustrate the correlation between the actual and the predicted A $\beta-P E T$ zscore when using fibrillar $A \beta$ and non-fibrillar $A \beta$ as predictors, and individual heterogeneity and age as covariates. Regressions were calculated with a total of $n=261$ permutations between immuno/histochemistry and PET endpoints using all available combinations with matched age and genotype. (D) Application of the average regression factors for fibrillar $(B=3.17)$ and non-fibrillar $A \beta(B=0.20)$ on the combined immuno/histochemistry data of both models (group means per age). 

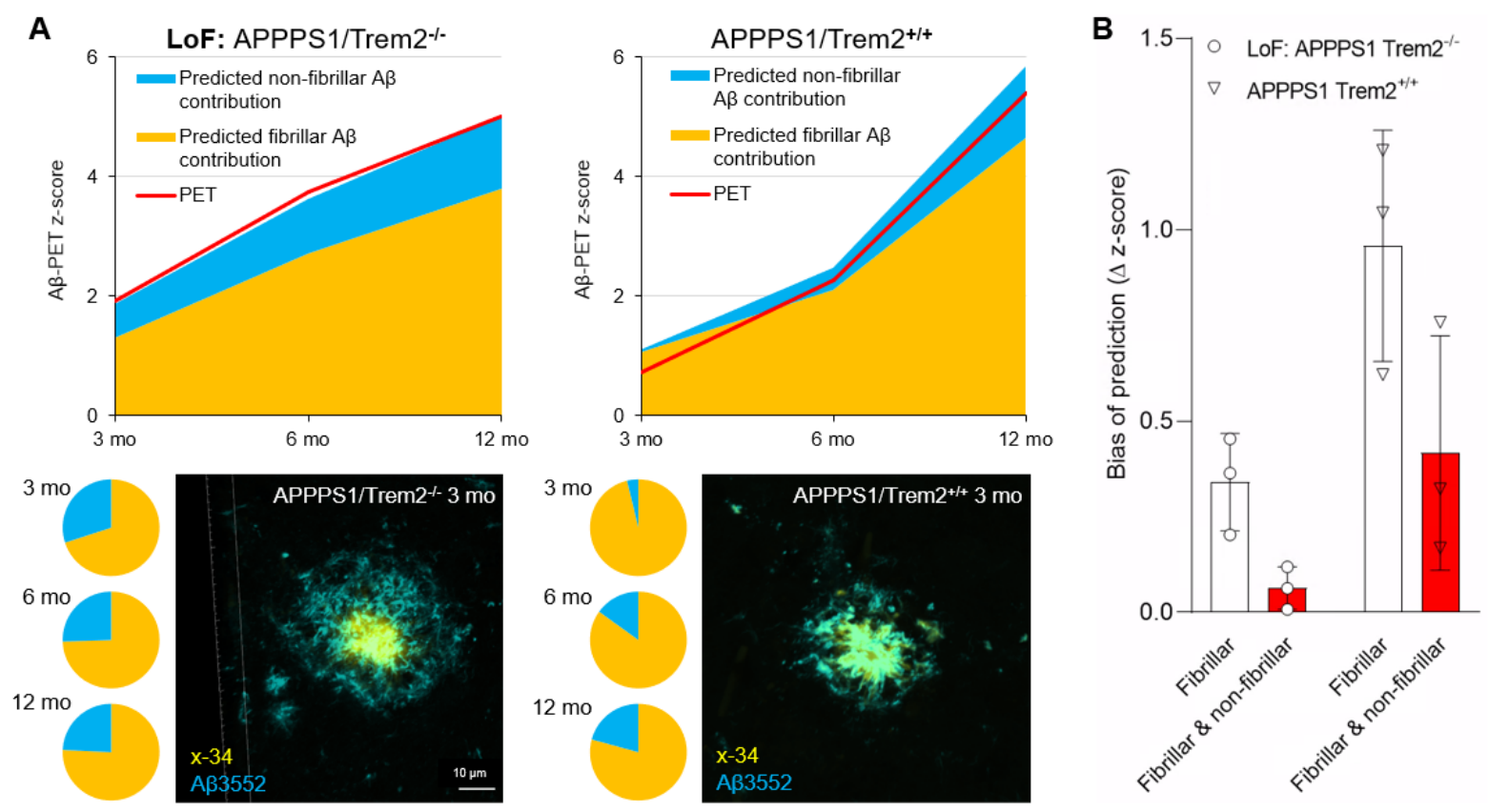

Figure 4: $(A) Z$-scores of the measured $A \beta$-PET signal (red) and the predicted fibrillar (orange) and nonfibrillar (blue) source components in an independent cohort of APPPS1 mice with dysfunctional (Trem2 $2^{-1-}$ ) and intact $\left(\right.$ Trem $\left.^{+/+}\right)$microglia. Pie charts show the fibrillar and non-fibrillar contributions to the measured $A \beta-P E T$ signals predicted by immuno/histochemistry. A representative double staining of a APPPS1/Trem21- mouse shows more non-fibrillar $A \beta$ (3552-positive, blue) surrounding the core ( $x$-34-positive, yellow/orange) when compared to a APPPS1/Trem $2^{+/+}$mouse, both at three months of age. (B) Bias of the predicted z-scores, when only fibrillar or the combination of fibrillar and non-fibrillar plaque contributions were considered. The analysis displays the deviation of predicted values and the actual A $\beta$-PET signal in part $B$ at the three different ages. 


\begin{tabular}{|l|c|c|c|c|c|}
\hline \multicolumn{1}{|c|}{ Model } & $R^{2}$ & $R^{2}$ (corr.) & $\begin{array}{c}\text { Reg. coeff } \\
\text { Fibrillar (B) }\end{array}$ & $\begin{array}{c}\text { Reg. coeff } \\
\text { Non-fibrillar (B) }\end{array}$ & $P$-value \\
\hline Fibrillar & 0.499 & 0.497 & $4.414(3.915-4.926)$ & & $P<0.001$ \\
\hline Non-fibrillar & 0.324 & 0.322 & & $0.477(0.378-0.569)$ & $P<0.001$ \\
\hline Fibrillar x non-fibrillar & 0.574 & 0.571 & $3.521(2.954-4.041)$ & $0.259(0.178-0.341)$ & $P<0.001$ \\
\hline $\begin{array}{l}\text { Fibrillar X non-fibrillar } \mathrm{X} \\
\text { heterogeneity }\end{array}$ & 0.854 & 0.852 & $3.521(2.954-4.041)$ & $0.259(0.178-0.341)$ & $P<0.001$ \\
\hline $\begin{array}{l}\text { Fibrillar X non-fibrillar } \mathrm{x} \\
\text { heterogeneity x age }\end{array}$ & 0.928 & 0.927 & $2.810(2.620-3.032)$ & $0.146(0.103-0.193)$ & $P<0.001$ \\
\hline
\end{tabular}

Table 1: Coefficients of determination $\left(R^{2}\right)$ and regression coefficients $(B)$ are given for prediction of the $A \beta-P E T$ signal by fibrillar and non-fibrillar $A \beta$ components, with additional factoring for heterogeneity (in mice with equal genotype at a single time-point) and age. Numbers in brackets represent the $95 \%$-confidence interval as assessed by bootstrapping with 1000 random samples. 


\section{Supplement}

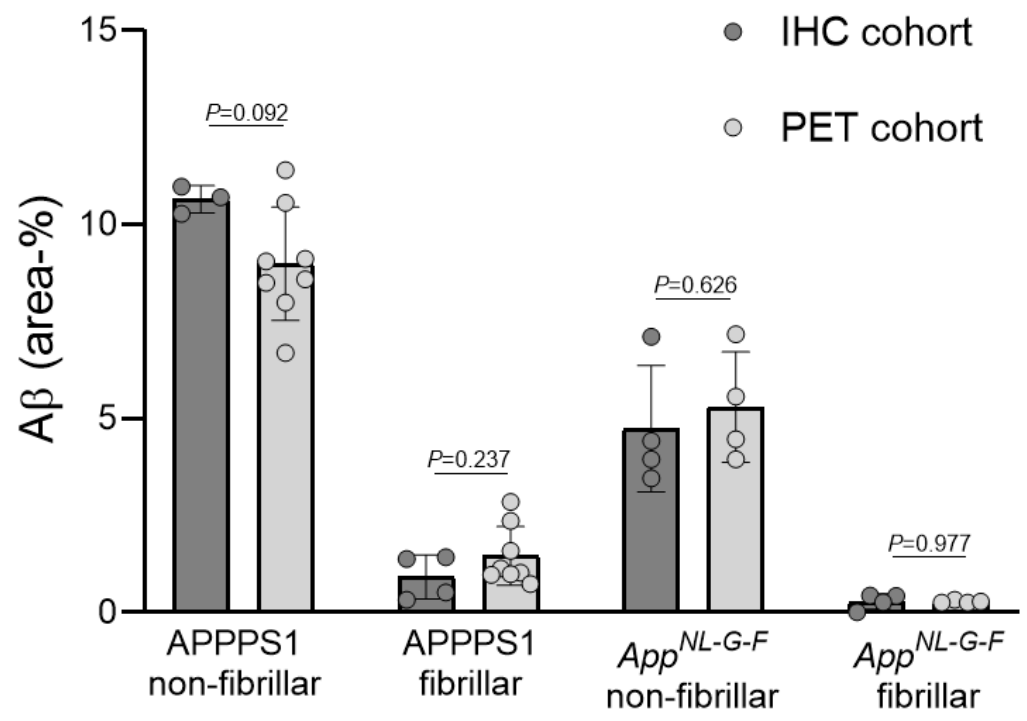

Supplemental Figure 1: Comparison of $A \beta$ area coverage of the immunohistochemistry (IHC) cohort and the PET cohort for both $A \beta$ mouse models. Note that different histology markers were used for assessment of fibrillar $A \beta$ (Thiazine red, $x-34$, methoxy-x04). Non-fibrillar $A \beta$ was consistently assessed by 3552. P-values derive from a Student's $t$-test. Error bars represent SD. 

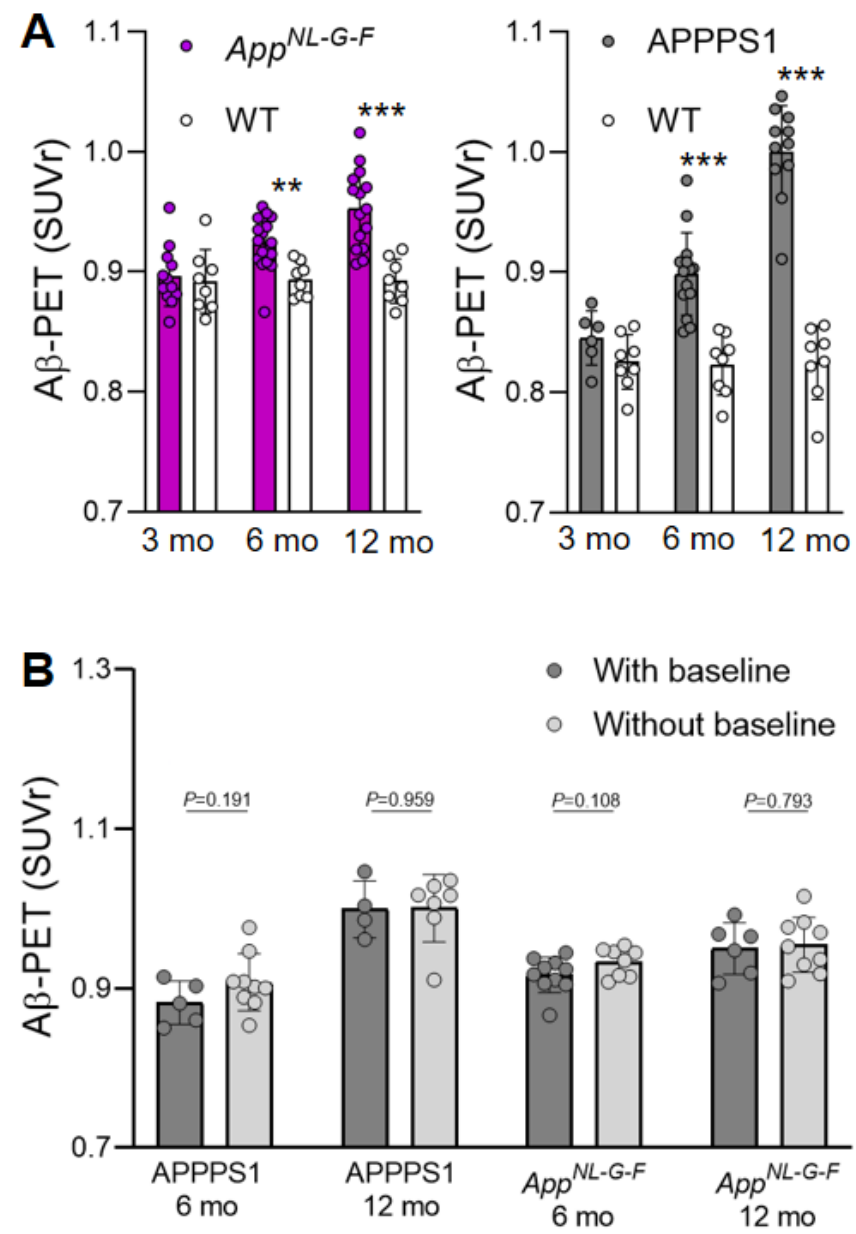

Supplemental Figure 2: (A) PET SUVr quantification in A $\beta$ mouse models and wild-type (WT) mice. Previously validated model-specific periaqueductal grey and white matter reference tissues were used for SUVr calculation. Z-score calculation of these SUVr values (TG - WT mean / WT enabled the direct comparison of both $A \beta$ mouse models in the regression models of the main manuscript. (B) Comparison of A $\mathrm{B}$-PET standardized uptake value ratios (SUVr) between mice with baseline examination at 3 months compared to mice with baseline examination at 6 months. $P$-values derive from a Student's $t$-test. ${ }^{* *} P<0.01 ;{ }^{* *} P<0.001$. Error bars represent SD. 


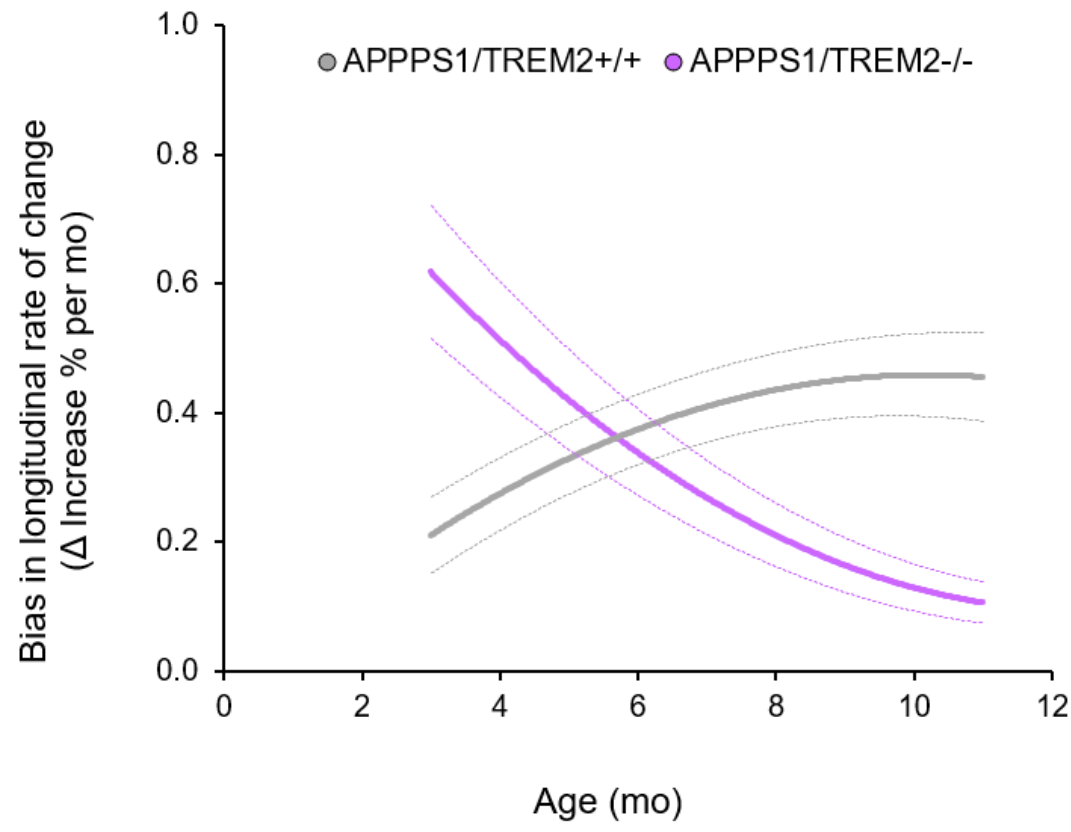

Supplemental Figure 3: Bias of $A \beta-P E T$ rate of change when fibrillar $A \beta$ is considered soley. Fits represent polynomic functions of the bias in longitudinal changes of A $\beta$-PET in APPPS1/Trem2 ${ }^{-/-}$ mice (purple) in contrast to APPPS1/Trem2 ${ }^{+/+}$mice (grey). Dotted lines represent functions of s.e.m. The plot was created analogous to (Parhizkar, Arzberger et al. 2019). 